\title{
Use of procalcitonin as a tool for antibiotic stewardship
}

\author{
Rita Murri ${ }^{1}$, Eleonora Taddei ${ }^{1}$, Roberto Cauda*,1 \& Massimo Fantoni ${ }^{1}$ \\ ${ }^{1}$ Department di Clinica delle Malattie Infettive, Università Cattolica S Cuore, Fondazione Policlinico A Gemelli IRCCS, Roma, Italy \\ *Author for correspondence: Tel.: +39 3015 4945; Roberto.cauda@unicatt.it
“PCT dosing helps to reduce antibiotic therapy duration for serious infections - with no harm in terms of mortality"

First draft submitted: 23 November 2018; Accepted for publication: 4 December 2018; Published online: 15 January 2018

Keywords: acute infections $\bullet$ antimicrobial stewardship • intensive care unit $\bullet$ procalcitonin $\bullet$ sepsis

Since the first observations of its elevation as a result of inflammatory state, notably in cases of bacterial infection [1], procalcitonin (PCT) has been extensively studied and recognized as a valuable biomarker to support diagnosis and guide therapy in infectious diseases. Even after some shortcomings emerged - especially due to a low microbiological predictive power [2] - PCT retained its clinical value, especially after recent redefinition of sepsis as a clinical syndrome, due to its correlation with the SOFA score and with patient outcomes [3].

After more than 20 years of study, most evidence regarding PCT role and its potential comes from the intensive care unit (ICU) setting. Fewer data were produced in the field of primary care, internal medicine, and in the emergency department [4].

Antimicrobial stewardship (AS) approach, as proposed by major guidelines [5], must be considered a cornerstone of clinical practice for the purpose of promoting optimization of the use of antimicrobial regimens, with the aim of limiting multidrug resistance spread. In this regard, PCT-guided de-escalation or discontinuation of antibiotics, which are supported by extensive literature and already endorsed by local and international guidelines [6,7], perfectly fits in such strategy.

As for any biomarker in medicine, misuse may lead to a high volume of useless determinations. This in turn, can lead to perception of limited utility of PCT by clinicians and bears the risk of wasting its potential, to support saving policies in hospitals. An optimal use of PCT measurement may contribute to improve patient management and saving resources. It is, therefore, of the greatest importance to preserve PCT as a tool in the AS setting by means of correct use. The aim of this commentary is to discuss research fields that could be further investigated to improve the use of PCT, in the context of an AS approach.

We propose four areas for which further studies are strongly warranted to define optimal PCT utilization in clinical settings, outside of the ICU: selection of patients, definition of appropriate cut-off integration with microbiological data and inclusion in an AS approach.

\section{Patient selection}

As it is the case with any test in medical practice, the value of results highly depends on pretest considerations aimed at identifying patients who would take greatest benefit from being tested. More specifically, predictive value of a test is correlated to the pre-test prevalence of the disease. Most relevant data on correlation of PCT elevation to infection are available for bloodstream infections (BSI), sepsis and pneumonia; most data come from the ICU. Therefore, it would be very useful to identify study populations in whom suspicion of BSI, sepsis or pneumonia is high. Currently, clinical risk scores, such as SOFA for sepsis [3] and CURB-65 or PSI for pneumonia [6] are validated to predict severity of the disease. The predictive value of PCT in patients stratified according to these scores, could improve the performance of the measurement.

On the other hand, elevation during other infections (i.e., endocarditis and meningitis) is more variable, hence it is difficult to interpret PCT results [8,9]. Moreover, escalation strategies based on PCT-guided approaches are not

Future 8 Medicine 
validated [10]. Further studies on the meaning of high PCT levels in patients for whom an infectious disease has been ruled out is another research area of great interest.

\section{Predefinition of cut-offs}

Since most data on PCT utilization come from intensive care settings, the performance of PCT measurement in medical wards should be further investigated. A highly accepted cut-off of $<0.5 \mathrm{ng} / \mathrm{ml}$ is used to discourage both infectious diagnosis and antibiotic therapy initiation in ICU clinical trials $[11,12]$. Whether the same cut-off could be adopted for lower-intensity care settings, should be evaluated. Particularly, a cut-off of $1 \mathrm{ng} / \mathrm{ml}$ or even higher could increase the test specificity in non-ICU settings. As no large agreement exists on an algorithm for PCT measurement, quality evidence is needed to guide appropriate use. In the meantime, we suggest that AS teams in healthcare facilities provide rules on timing of determinations and interpretation of cut-off.

\section{Integration with microbiology data}

PCT should not be regarded as an alternative to rapid microbiological tests, nor as a tool to rule out BSI. Neither should it discourage blood cultures collection in case of negativity, since its negative predictive value for bacteremia is poor [2]. A decreasing trend of elevation exists from Gram-negative bacteria, to Gram-positive bacteria, to fungi [13]. PCT elevation proved useful if combined with low $\beta$-D-glucan levels to hint against fungal infections [14]. Whether PCT measurement may accelerate the identification of the cause of an infection, could be another interesting field to be investigated.

\section{Antimicrobial stewardship approach}

It has been largely demonstrated that the greatest utility of PCT is in guiding antibiotic de-escalation, based on PCT reduction. PCT dosing helps to reduce antibiotic therapy duration for serious infections - with no harm in terms of mortality $[10,11]$. It is not surprising that PCT potential to reduce length of therapy was also studied in terms of health economics, showing expenditure reduction [15]. A reduction in antimicrobial utilization is correlated to a reduced selective pressure on local microbiota.

Providing AS teams with PCT levels along with other data can improve patient care and optimize antimicrobial utilization with fewer negative effects both at patient and environmental level. Pharmacoeconomic studies on the benefits of PCT measurement in clinical practice, in different settings and different populations are strongly warranted.

In conclusion, we believe that PCT measurement, when applied to the right patient population and in the context of AS programs, can prove useful in reducing inappropriate antimicrobial therapies. We suggest that all clinicians comply with international or local guidelines, if available, such that PCT determination can be limited to appropriate cases, always estimating in advance if a positive or negative result will have a consequence in terms of clinical practice.

Further data from settings outside of ICUs are strongly needed to support infectious disease specialists and antimicrobial stewards in critically revising local PCT protocols and promoting a wise use of this biomarker. PCT is a useful weapon in the AS arsenal against antimicrobial resistance and should not be wasted.

Financial \& competing interests disclosure

The authors have no relevant affiliations or financial involvement with any organization or entity with a financial interest in or financial conflict with the subject matter or materials discussed in the manuscript. This includes employment, consultancies, honoraria, stock ownership or options, expert testimony, grants or patents received or pending, or royalties.

No writing assistance was utilized in the production of this manuscript.

\section{References}

Papers of special note have been highlighted as: $\bullet$ of interest; $\bullet \bullet$ of considerable interest

1. Assicot M, Gendrel D, Carsin H et al. High serum procalcitonin concentrations in patients with sepsis and infection. Lancet 341(8844), 515-518 (1993).

2. Thomas-Rüddel D, Poidinger B, Kott M et al. Influence of pathogen and focus of infection on procalcitonin values in sepsis patients with bacteremia or candidemia. Crit. Care 22(1), 128 (2018).

3. Singer M, Deutschman C, Warren Seymour C et al. The third international consensus definitions for sepsis and septic shock (Sepsis-3). JAMA 315(8), 801-810 (2016). 
•- The Consensus on the 2016 new definition of sepsis and the recommendations for the managemnt of patients with sepsis

4. Schuetz P, Bretscher C, Bernasconi L, Mueller B. Overview of procalcitonin assays and procalcitonin guided protocols for the management of patients with infections and sepsis. Expert Rev. Mol. Diagn. 17(6), 593-601 (2017).

5. Barlam TF, Cosgrove SE, Abbo LM et al. Implementing an Antibiotic Stewardship Program: guidelines by the Infectious Diseases Society of America and the Society for Healthcare Epidemiology of America. Clin. Infect. Dis. 62(10), e51-e77 (2017).

6. Woodhead M, Blasi F, Ewig S et al. Guidelines for the management of adult lower respiratory tract infections. Clin. Microbiol. Infect. 17, E1-E59 (2011).

7. Rhodes A, Evans L, Alhazzani W et al. Surviving sepsis campaign guidelines. Intensive Care Med. 43(3), 304-377 (2017).

8. Yu CW, Juan LI, Hsu SC et al. Role of procalcitonin in the diagnosis of infective endocarditis: a meta-analysis. Am. J. Emerg. Med. 31(6), 935-941 (2013).

9. Vikse J, Henry BM, Roy J, Ramakrishnan PK, Tomaszewski KA, Walocha JA. The role of serum procalcitonin in the diagnosis of bacterial meningitis in adults: a systematic review and metanalysis. Int. J. Infect. Dis. 38, 68-76 (2015).

10. Jensen JU, Hein L, Lundgren B et al. Procalcitonin-guided interventions against infections to increase early appropriate antibiotics and improve survival in the intensive care unit: a randomized trial. Crit. Care Med. 39(9), 2048-2058 (2011).

11. Bouadma L, Luyt CE, Tubach F et al. Use of procalcitonin to reduce patients' exposure to antibiotics in intensive care units (PRORATA trial): a multicentre randomized controlled trial. Lancet 375(9713), 463-474 (2010).

\section{- A large randomized clinical trial on an algorithm for driving antibiotic management according to PCT levels}

12. de Jong E, van Oers JA, Beishuizen A et al. Efficacy and safety of procalcitonin guidance in reducing the duration of antibiotic treatment in critically ill patients: a randomised, controlled, open-label trial. Lancet Infect. Dis. 16(7), 819-827 (2016).

13. Murri R, Mastrorosa I, Taccari F et al. Procalcitonin is useful in driving the choice of early antibiotic treatment in patients with bloodstream infections. Eur. Rev. Med. Pharmacol. Sci. 22(10), 3130-3137 (2018).

14. Giacobbe D, Mikulska M, Tumbarello $\mathrm{M}$ et al. Combined use of serum (1,3)- $\beta$-D-glucan and procalcitonin for the early differential diagnosis between candidaemia and bacteraemia in intensive care units. Crit. Care 21(1), 176 (2017).

15. Westwood M, Ramaekers B, Whiting P et al. Procalcitonin testing to guide antibiotic therapy for the treatment of sepsis in intensive care settings and for suspected bacterial infection in emergency department settings: a systematic review and cost-effectiveness analysis. Health Technol. Assess. 19(96), 1-236( 2015). 
